\title{
One-day cognitive-behavioural therapy self-confidence workshops for people with depression: randomised controlled trial
}

Linda Horrell, * Kimberley A. Goldsmith,* André T. Tylee, Ulrike H. Schmidt, Caroline L. Murphy, Eva-Maria Bonin, Jennifer Beecham, Joanna Kelly, Shriti Raikundalia and June S. L Brown on behalf of the CLASSIC trial group

\section{Background}

Despite its high prevalence, help-seeking for depression is low.

\section{Aims}

To assess the effectiveness and cost-effectiveness of 1-day cognitive-behavioural therapy (CBT) self-confidence workshops in reducing depression. Anxiety, self-esteem, prognostic indicators as well as access were also assessed.

\section{Method}

An open randomised controlled trial (RCT) waiting list control design with 12-week follow-up was used (trial registration: ISRCTN26634837). A total of 459 adult participants with depression (Beck Depression Inventory (BDI) scores of $\geqslant 14$ ) self-referred and 382 participants (83\%) were followed up.

\section{Results}

At follow-up, experimental and control participants differed significantly on the BDI, with an effect size of 0.55 . Anxiety and self-esteem also differed. Of those who participated, $25 \%$ were GP non-consulters and 32\% were from Black and minority ethnic groups. Women benefited more than men on depression scores. The intervention has a $90 \%$ chance of being considered cost-effective if a depression-free day is valued at $f 14$

\section{Conclusions}

Self-confidence workshops appear promising in terms of clinical effectiveness, cost-effectiveness and access by difficult-to-engage groups.

\section{Declaration of interest}

None.
By 2030, depression is forecast to be the leading cause of disease burden worldwide. ${ }^{1}$ Despite its high prevalence rates, numerous barriers prevent seeking and accessing help so that depression remains vastly undertreated. $^{2}$ In the UK, $54 \%$ of people experiencing a depressive episode did not contact their general practitioner (GP). ${ }^{3}$ In addition, although the public prefer psychological treatment over medication for depression, ${ }^{4}$ psychological services have been very limited. ${ }^{5}$ In the UK, access to psychological therapies is currently mostly via referral from a GP or a health professional. Despite a recent increase in funding, the capacity of psychological treatment services remains limited. In addition, Black and minority ethnic (BME) groups are often underrepresented in psychological therapy services, ${ }^{6}$ as shown in the demonstration sites for the Improving Access to Psychological Therapies (IAPT) initiative in the UK. ${ }^{7}$

Cognitive-behavioural therapy (CBT) is as effective as medication in individuals with moderate to severe depression, and has long-term benefits. ${ }^{8}$ Individual and group CBT for depression have comparable effectiveness. ${ }^{9}$ Clinical guidelines in the UK recommend intensive individual CBT for those with moderate or severe depression, whereas individuals with mild to moderate depression who decline first-line low-intensity treatments (e.g. computerised CBT and guided self-help) should be offered group CBT. ${ }^{10}$ Traditional group CBT tends to be small scale, with $8-10$ participants meeting for $10-122 \mathrm{~h}$ sessions. A credible alternative is to offer larger-scale psychoeducational CBT groups that can reach more people. This approach has been successfully used with primary care patients with generalised anxiety who were offered evening classes ${ }^{11}$ and members of the public who self-referred to 1 -day stress workshops. ${ }^{12}$ However,

*Joint first authors. psychoeducational interventions advertised as 'depression' workshops had a lower uptake, attracting mostly people who had already used specialist services. ${ }^{13}$ Changing the name of the workshops to a non-diagnostic label of 'self-confidence' workshop led to a much higher uptake, with $39 \%$ of self-referrers never having previously consulted their GP for depression. ${ }^{14}$ A small randomised controlled trial (RCT) of 1-day self-confidence workshops v. a waiting list control found the intervention to be effective in reducing depression and improving self-esteem after 12 weeks. A naturalistic follow-up study found that the benefits were maintained at 2 years but only for those who were depressed. ${ }^{15}$ So far, the effectiveness of these brief workshops has only been demonstrated with a group of people varying in depression symptoms in one relatively deprived part of London, and no full economic evaluation has been undertaken. This study aims to assess whether the self-confidence workshops can be effective and cost-effective in areas with different deprivation levels, focusing just on people with depression. If shown to be successful, this could provide an alternative effective and costeffective psychological intervention for people with depression in the community, given the low take-up rates for treatment for depression and preferences for psychological treatment.

\section{Method}

\section{Design}

A multicentre open RCT design was used, with self-confidence workshops run across eight boroughs in south London, with experimental and waiting list control arm participants followed up after 12 weeks. Workshops were run between April 2010 and July 2011. Ethical approval was obtained from the King's College Ethical Committee (Ref: PNM/09/10-65). 
The aims of the study were:

(a) to assess whether the self-confidence workshops affected depression, the primary clinical outcome;

(b) to assess the effect of the workshops on the secondary clinical outcomes of anxiety and self-esteem;

(c) to investigate prognostic indicators of those who benefit most from the workshops;

(d) to assess the proportion of participants from difficult-toengage groups that accessed the intervention, specifically GP non-consulters and BME groups, in relation to local population distributions;

(e) to assess whether the workshops were cost-effective compared with treatment as usual.

\section{Study setting}

The study was run in eight London boroughs. Using rank scores (range 1-326, where 1 indicates most deprived) derived from the Index of Multiple Deprivation 2010, ${ }^{16}$ in order of decreasing deprivation, the boroughs were: Greenwich (28), Lambeth (29), Lewisham (31), Croydon (107), Wandsworth (121), Bexley (174), Merton/Sutton (208), and Kingston upon Thames (255). Because of initial excess attrition, Croydon and Lewisham were both revisited for a second workshop to ensure sufficient statistical power for outcome comparisons.

Because workshops were designed to be accessible to the community, a self-referral process was used to recruit participants. Publicity was distributed for workshops 2-3 months before each introductory talk, which is where participants were recruited. A5 flyers advertising free 1-day workshops entitled 'How to improve your self-confidence' were posted to libraries, GP practices, community centres, leisure centres and pharmacies. The same advertisement was displayed in local magazines a few weeks before the introductory talk. Interested individuals were asked to email or telephone for further information and to register for the introductory talk.

To maximise access, all workshops were held on Saturdays in non-mental health settings such as libraries, community centres or leisure centres. Venues also had good public transport links and disabled access.

\section{Participants}

Study participants had to be at least 18 years of age and have depression, as indicated by a Beck depression Inventory ${ }^{17}$ score of 14 or above. Other exclusion criteria are given in the CONSORT diagram (Fig. 1) and included unavailability for the workshop dates and people attending the workshop together (because of the possibility of 'contamination' if they were randomised to different groups). We also excluded those who were unable to complete the baseline self-assessment questionnaires as well as, under 'other', those participants who had attention and concentration difficulties during the introductory talk. Research assistants and/or workshop leaders assessed participants against these criteria when registering or at the introductory talks. No exclusion criteria were specified in relation to antidepressants or concurrent psychological therapy. For ethical reasons, individuals who were not eligible or did not want to participate in the study

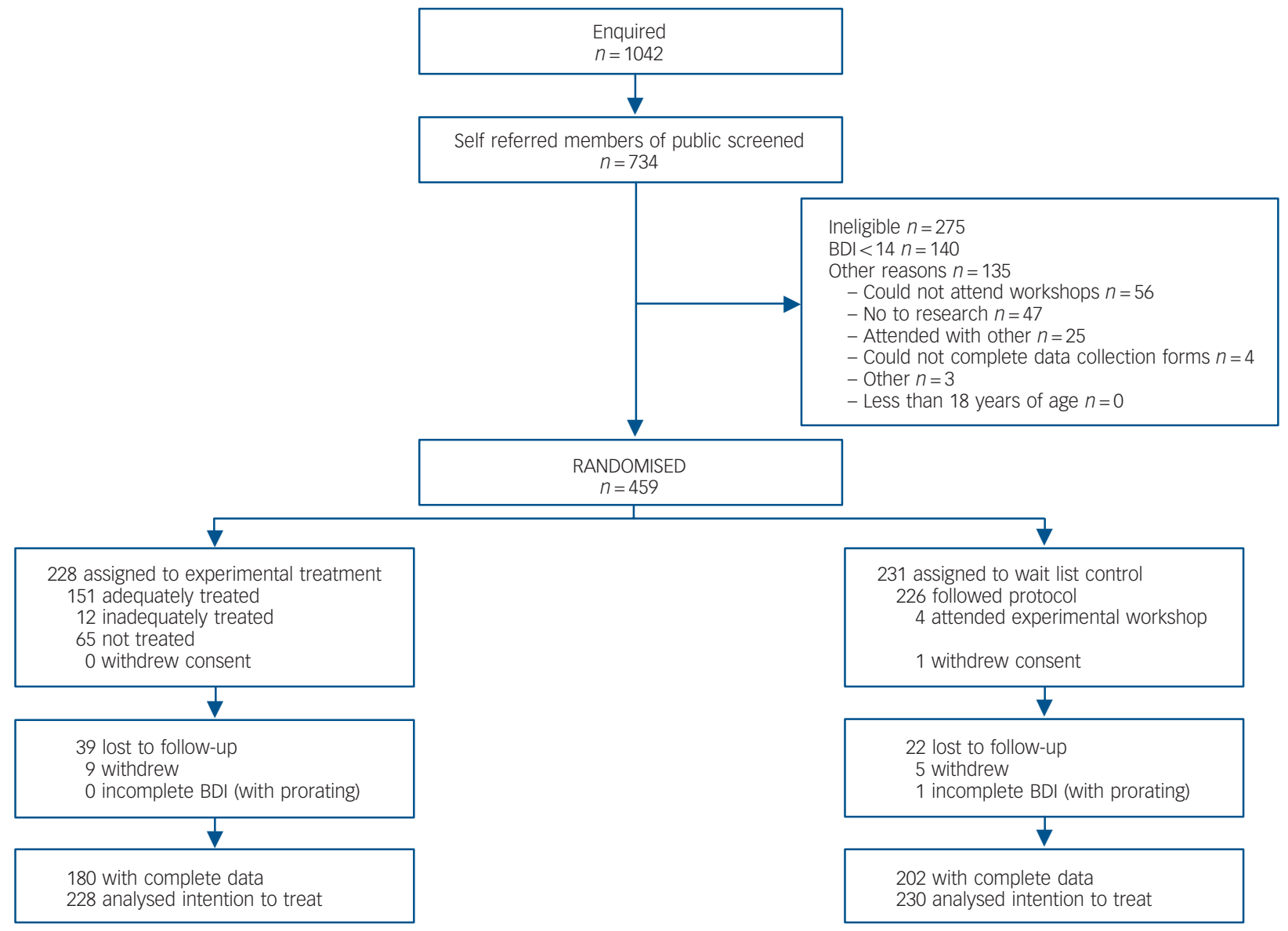

Fig. 1 Flow of participants through the trial. BDI, Beck Depression Inventory. 
were invited to attend workshops held on different dates to the study workshops.

\section{Intervention}

The psychoeducational self-confidence workshop programme adopted for this study followed the previously piloted programme ${ }^{14}$ and used cognitive-behavioural techniques largely based on Fennell's model. ${ }^{18}$ Up to 30 people could attend each workshop. The day's programme ran from $09.30 \mathrm{~h}$ to $16.30 \mathrm{~h}$ and was structured into four sessions. First, information was given about the development of low self-confidence and its emotional components, including depression. The second session consisted of cognitive components of low self-confidence, particularly identifying and challenging negative thoughts. Behavioural methods for improving low self-confidence, including problemsolving and assertiveness, were taught in the third session. The final session was devoted to action planning, with participants setting their own homework targets to start improving their confidence. To reduce the possibility of participants becoming bored or tired, training methods were varied and included didactic sections, large group exercises, small group exercises, role-play demonstrations and discussions of vignettes of people with low self-confidence. Each participant was given a colourful 54-page A4 workbook that covered the day's programme to remind them of the methods taught. One month after the experimental arm workshop, participants were invited to a nontherapeutic $2 \mathrm{~h}$ booster session run by the same workshop leaders to help consolidate learning.

Workshops were run by two teams, each comprising two clinical or counselling psychologists, with two reserve psychologists providing cover in case of unavailability. Workshop leaders had an average of 3.5 years' (range 2-7) post-qualification experience in delivering CBT. Workshop leaders received training in delivering the programme by undergoing a 2-day training programme, in which they observed a workshop and then ran a workshop themselves under observation on a single day. The programme was manualised to ensure standardisation across boroughs. Workshop leaders also had bimonthly supervision, led by J.S.L.B., to review any difficult situations.

\section{Measures}

Self-report questionnaires were administered at the introductory talk and 12 weeks after randomisation and comprised the following measures.

\section{Primary outcome measures}

Beck Depression Inventory-Second Edition (BDI-II, referred to here as BDI): ${ }^{19}$ to measure severity of depression. Scores are normally categorised into non-depressed (score $<10$ ), mildly depressed (10-19), moderately depressed (20-28) and severely depressed $(\geqslant 29)$. An eligibility criterion used for this study was a BDI score of 14 and above. ${ }^{17}$ Cronbach's alpha is 0.92 for clinical patients and 0.93 for non-clinical individuals. ${ }^{19}$ For the economic evaluation, depression-free days (DFD) were calculated based on BDI scores at follow-up, using an algorithm suggested by Lave et al. ${ }^{20}$ A full DFD was assigned for BDI $<10$ and no DFD for BDI $>28$, with scores in between weighted proportionally. The maximum number of DFD over a 3-month period was 91 .

\section{Secondary outcome measures}

Generalised Anxiety Disorder-7 (GAD-7). A seven-item, fourpoint scale to assess anxiety. Cronbach's alpha is $0.92 .{ }^{21}$
Rosenberg Self-Esteem Scale (RSES). A ten-item, four-point scale to measure changes in self-esteem. Cronbach's alphas vary from 0.77 to $0.88 .{ }^{22}$

EQ-5D. A measure of health-related quality of life. ${ }^{23}$ Health status is measured on five dimensions on three-point scales. The associated tariffs for England ${ }^{24}$ are then applied to calculate quality-adjusted life-years (QALYs). The instrument also includes a visual analogue scale, allowing individuals to rate their current health status on a scale from 0 (worst) to 100 (best).

Client Service Receipt Inventory (CSRI). ${ }^{25}$ A self-report version of the measure, covering a retrospective 3 -month period, was adapted for the study to record use of services including hospital, primary care, specialist mental health and community-based services such as social work and alternative therapy. It also records demographic information and details on employment to measure societal costs. In addition, it records whether GPs had prescribed antidepressants for participants.

Client Satisfaction Questionnaire (CSQ) ${ }^{26}$ This was completed at the end of each workshop, This eight-item scale measures satisfaction on a four-point scale. Cronbach's alpha for the scale is $0.92-0.93 .^{27}$

\section{Procedure}

All individuals who registered were invited to a $1 \mathrm{~h}$ group introductory talk where further information about the workshops was given and informed consent obtained before participants completed baseline assessments. For those people who were unavailable for the talk, the research assistants offered an individual telephone assessment in the week either prior to or after the introductory talk. Eligible participants were randomly allocated either to receive the workshop after 3 weeks (experimental arm) or to wait for 12 weeks for the workshop (control arm). One month after the experimental arm workshop, participants were invited to a non-therapeutic $2 \mathrm{~h}$ booster group session run by the same workshop leaders to help consolidate learning. At follow-up, a $2 \mathrm{~h}$ group meeting was held for experimental arm participants when outcome measures were completed at the beginning of the session, followed by a group discussion and signposting to other services as appropriate. Participants in the control arm were advised to see their GP as usual during the 12-week waiting period. At 12-week follow-up, outcome measures for the control arm were collected immediately before they attended the workshop.

Study participants who did not complete the assessments were posted outcome assessment questionnaires. This was followed up by telephone contact up to five times, as necessary. If there was no response, a final shortened assessment pack was posted.

\section{Sample size}

The calculated sample size for $90 \%$ power to detect an effect size of 0.46 was 320 , or 160 individuals in each arm. This was calculated assuming a 5\% significance level, a two-sided test, an attrition rate of $15 \%$, and given the group intervention, a cluster size of 20 and an intraclass correlation coefficient of 0.05. Because the attrition level was higher than expected in Lewisham, Wandsworth and Merton/Sutton (the first sites to be studied) (32\%), the target sample size was increased to 420 (210 per arm) to ensure adequate power. 


\section{Randomisation and masking}

Eligible participants were randomly assigned to one of the two treatment arms after baseline assessments were completed and consent obtained. Randomisation was performed using an online randomisation system provided by the King's Clinical Trials Unit at the Institute of Psychiatry, King's College London. Randomisation was stratified by gender, self-reported ethnicity (White/Other), depression (BDI mild, moderate and severe categories) and borough. Minimisation was used to reduce any imbalance on these features across the two arms. Simple randomisation was used for the first 32 participants and the minimisation algorithm utilised for the subsequent 427 participants. Research assistants electronically submitted details of each participant to the Clinical Trials Unit and the randomisation outcome was immediately returned electronically. An open design was used as masking of arm allocation for research assistants and participants was not possible. However, the trial statistician's masking was maintained until the primary analysis.

\section{Statistical methods}

Stata version 10 for Windows was used for the main analysis. Variables were described using frequency and proportion or mean and standard deviation as appropriate.

\section{Participant characteristics}

Categorical variables were compared using Chi-squared and Fisher's exact tests. Continuous data were analysed using $t$-tests. Chi-squared goodness of fit tests were used to compare our sample with the local population for each borough using information from the Office for National Statistics. ${ }^{28}$

\section{Clinical outcome analysis}

Clinical outcomes analysis was carried out following intention-totreat (ITT) principles. Incomplete baseline and outcome BDI, GAD-7 and RSES questionnaires were prorated when $20 \%$ or less of the items were missing, with the mean value of other responses used to replace the missing response. Categorical variables were compared using Fisher's exact test.

Adequate treatment completion was defined as experimental arm participants attending the full day-long workshop and control arm participants not attending the earlier experimental workshop. A logistic regression model with an indicator variable for missing outcome data as the dependent variable was used to examine whether treatment non-completion and other variables predicted missing outcome. As this was the case, multiple imputation using chained equations was applied (Stata mi and mim commands). ${ }^{29}$ Imputation allows for the inclusion of post-treatment variables that predict missing data, including completion of treatment, and tends to be a more conservative analysis method. The imputation model covariates included an indicator variable for treatment completion, other variables associated with missing data, treatment arm, stratification factors (gender, ethnicity and borough), baseline and follow-up values of primary and secondary outcomes, and variables of interest for other secondary analyses. Thirty data-sets were imputed and Rubin's rules employed to obtain estimates. $^{30}$ A sensitivity analysis for the multiple imputation using a missing-at-random assumption examined the effect of adding a range of possible deviations to the ITT effect. $^{31}$ As higher BDI scores indicate increased depression, deviation values which equated to the missing values being between 2 and 20 points higher than the observed values in the experimental arm were tested. The analysis allowed the level of deviation at which the ITT effect would become non-significant to be quantified and the plausibility of such a deviation value to be evaluated.

To assess differences in outcome, linear regression models were used. Mean differences between the treatment arms and associated 95\% confidence intervals (CIs) for the primary and secondary outcomes were estimated with the pertinent measure at follow-up as the dependent variable, with treatment arm, baseline score of the measure, and the other stratification factors as covariates. Confidence intervals for parameters were estimated using robust standard errors to account for clustering by workshop.

The adjusted effect size was calculated as the mean difference between the two arms divided by the average root mean square error from models fitted to each imputed data-set.

Additional analyses included 'per protocol analysis', testing for an effect of therapist learning on BDI outcome over time and an exploration of baseline predictors of outcome BDI, using the imputed data. The per protocol analysis included only the treatment completers. The test for a trend was done using experimental arm data only and a continuous fixed effect for workshops numbered sequentially in time. The prognostic analysis explored whether various variables measured at baseline were predictive of outcome BDI. Manual forward stepwise regression was used to add variables to the base ITT analysis model; any variables with Wald $F$-test $P$-values of 0.2 or less when entered singly were considered further, and the variable with the smallest $P$-value was added first. The process was halted when no further variables had a Wald $F$-test $P$-value of less than 0.05 . Interaction terms between each of the potential prognostic variables that had been entered into the model and treatment arm were used to assess whether the treatment effect was moderated by any of these variables. Interaction terms significant at $P<0.05$ were retained.

\section{Service costs and cost of the workshop intervention}

The costs of service use for each participant were calculated by identifying an appropriate unit cost and duration for each service contact reported on the CSRI and multiplying it by the number of contacts reported. Unit costs were drawn from publicly available sources (data.gov.uk/dataset/nhs-reference-costs-2010-11), ${ }^{32}$ taken from previous studies or estimated using an equivalent method. Where service contacts were reported but the number of contacts was missing, the mean for all people in contact with that particular service was entered. Medication costs are based on net ingredient costs from the British National Formulary (www.bnf.org).

The cost of providing the whole intervention (introductory talk, day-long workshop and booster session) was calculated from information provided by the research team. This included the cost of the venue, advertising, workshop materials, staff time (training, preparation, administration, delivering the intervention) and volunteer time. ${ }^{33}$ All costs are presented in 2010/11 prices.

\section{Cost-effectiveness and cost-utility analyses}

Stata version 12 for Windows was used for the economic analyses. The cost- effectiveness and cost-utility of the workshops were explored using seemingly unrelated regression, ${ }^{34}$ using the sureg command. Separate regression models were fitted for (a) costs at follow-up and (b) each of the outcome measures considered in the economic evaluation (change in BDI, additional DFDs, QALY gain) as the dependent variable. ${ }^{35}$

For each combination of cost and outcome, 10000 bootstrap replications of the treatment group difference in costs and outcomes were generated and incremental cost-effectiveness ratios were calculated by dividing costs by the outcome. The probability, presented as a percentage, that the intervention is cost-effective 
was derived by calculating the proportion of incremental costeffectiveness ratios that indicated a cost-effective outcome for a range of values a funder or society may place on an outcome (willingness to pay, WTP). Plotting this probability against the corresponding values of WTP results in a cost-effectiveness acceptability curve. ${ }^{36}$ The analysis of DFDs was used to illustrate differential cost-effectiveness for men and women.

Cost values for the sensitivity analysis were derived by varying the intervention cost based on different assumptions about attendance. The 'base case' reflected individuals' attendances as recorded by the researchers. A 'worst case' scenario was created by applying to all sites a cost based on the lowest proportion of workshop attendance ( $46 \%$ of people attending the introductory talk) and the lowest observed proportion of booster session attendance (7\%). A 'best case' scenario was created by assuming that the workshops were run at $100 \%$ capacity, with 30 participants attending the workshop and booster sessions. Costeffectiveness acceptability curves were drawn for all three scenarios for QALY gains and change in BDI.

\section{Results}

Of the 1042 individuals who enquired about the workshops, 734 were screened at the introductory talks or by telephone (Fig. 1).
Of these, $37 \%$ were excluded, the most common reason being that their BDI scores were less than 14. A total of 459 individuals were randomised into the experimental $(n=228)$ and control $(n=231)$ arms. Follow-up data were obtained for $83 \%$ of participants. Baseline characteristics of the participants were comparable between arms, except for a greater proportion being married in the control arm, in addition to differences in employment status (Table 1 and online Table DS1). Data on service use, required for the cost-effectiveness analysis, were available for 380 participants (179 participants in the experimental arm and 201 in the control arm). There were no significant differences between the full and the cost-effectiveness analysis sample.

\section{Participant characteristics}

In all eight boroughs, a higher percentage of women were recruited (80\%) compared with the distribution in the community (51\%). The average age of the recruited population was 44.1 years, similar to that across boroughs (44.0 years) $(P>0.05)$.

Across all boroughs, 25\% had never consulted their GP before for psychological help, with no differences by borough $(P=0.98)$.

Across the boroughs, there was a significant difference in the distributions across ethnic groups in the screened population compared with the borough population $\left(\chi^{2}=90.8\right.$, d.f. $=4$,

\begin{tabular}{|c|c|c|c|}
\hline & $\begin{array}{l}\text { Experimental group } \\
\qquad(n=228)\end{array}$ & $\begin{array}{l}\text { Control group } \\
\qquad(n=230)\end{array}$ & $\begin{array}{l}\text { Overall } \\
(n=458)\end{array}$ \\
\hline Age, years: mean (s.d.) & $42.3(12.1)$ & 45.9 (11.4) & $44.1(11.9)$ \\
\hline \multicolumn{4}{|l|}{ Ethnicity, n (\%) } \\
\hline Black & $37(16)$ & $30(13)$ & $67(15)$ \\
\hline White & $156(68)$ & $157(68)$ & $313(68)$ \\
\hline Asian & $23(10)$ & $25(11)$ & $48(11)$ \\
\hline Mixed & $8(4)$ & $14(6)$ & $22(5)$ \\
\hline Other & $4(2)$ & $4(2)$ & $8(2)$ \\
\hline \multicolumn{4}{|l|}{ Marital status, $n(\%)$} \\
\hline Single & $120(53)$ & $97(42)$ & $217(47)$ \\
\hline Married & $53(23)$ & $68(30)$ & $121(26)$ \\
\hline Cohabiting & $18(8)$ & $22(10)$ & $40(9)$ \\
\hline Separated & $7(3)$ & $14(6)$ & $21(5)$ \\
\hline Divorced & $26(11)$ & $26(11)$ & $52(11)$ \\
\hline Widowed & $4(2)$ & $3(1)$ & $7(2)$ \\
\hline \multicolumn{4}{|l|}{ Education, $n$ (\%) } \\
\hline O-level/GCSE & $49(22)$ & $58(25)$ & $107(23)$ \\
\hline A-level & $27(12)$ & $24(10)$ & $51(11)$ \\
\hline GNVQ & $22(10)$ & $30(13)$ & $52(11)$ \\
\hline University degree & $105(46)$ & $96(42)$ & 201 (44) \\
\hline None of the above & $25(11)$ & $22(10)$ & $47(10)$ \\
\hline \multicolumn{4}{|c|}{ Ever seen GP for psychological problems, $n$ (\%) } \\
\hline No & $59(26)$ & $53(23)$ & $112(25)$ \\
\hline Yes & $169(74)$ & $177(77)$ & $346(76)$ \\
\hline \multicolumn{4}{|c|}{ Tried counselling or psychological help before, $n$ (\%) } \\
\hline No & $54(24)$ & $44(19)$ & $98(21)$ \\
\hline Yes & $173(76)$ & $186(81)$ & $359(78)$ \\
\hline Missing & $1(0.4)$ & 0 & $1(0.2)$ \\
\hline \multicolumn{4}{|l|}{ Employment status, $n$ (\%) } \\
\hline Paid employment & $107(47)$ & $115(50)$ & $222(48)$ \\
\hline Volunteer work & $10(4)$ & $24(10)$ & $34(7)$ \\
\hline Unemployed & $64(28)$ & $41(18)$ & $105(23)$ \\
\hline Student & $9(4)$ & $4(2)$ & $13(3)$ \\
\hline Housewife/husband & $14(6)$ & $15(7)$ & $29(6)$ \\
\hline Retired & $14(6)$ & $22(10)$ & $36(8)$ \\
\hline Long-term sick/disabled & $10(4)$ & $9(4)$ & $19(4)$ \\
\hline EQ-5D utility score, mean (s.d.) & $0.6(0.3)$ & $0.6(0.3)$ & $0.6(0.3)$ \\
\hline
\end{tabular}


$P<0.001$ ) (Table 2). The proportion of Black participants selfreferring was more than 1.5 times that of the local population in five boroughs. Further, the proportion of Asian participants was more than twice that in the local population in three boroughs. Finally, the proportion of mixed ethnic groups was more than twice that in the community in five boroughs.

There was a significant association between ethnic group and level of GP consultation, with those of Black, Asian or other ethnic background more likely to be GP non-consulters than White or mixed ethnic groups $(P=0.003)$. The rate of GP consultation was lowest in the Asian ethnic group (60\%) and highest in the mixed ethnic group (82\%).

Severity of depression was also associated with level of GP consultation for depression. Significantly more people with severe depression (85\%) had previously consulted a GP than those with milder symptoms $(P<0.001)$. However, a post-hoc subgroup analysis by gender showed that the pattern held for women (mild depression, 59\%; moderate, $71 \%$; severe, $84 \%$; $P<0.001)$ but not for men (mild, $71 \%$; moderate, $69 \%$; severe, $88 \%$; $P=0.102$ ).

\section{Clinical outcomes}

Overall, 66\% of experimental arm and 98\% of control arm participants completed treatment (Fig. 1 and Table 3). The proportion of participants lost to follow-up was $8 \%$ greater in the experimental arm compared with the control arm (95\% CI $1-14, P=0.018$ ), but there was no significant difference between the arms in the proportion actively withdrawing from the trial (Fig. 1; $P=0.29$ ). After prorating, follow-up BDI was missing for $48(21 \%)$ experimental and $28(12 \%)$ control participants.

\begin{tabular}{|c|c|c|c|c|c|c|}
\hline Population & Black & White & Asian & Mixed & Other & Total \\
\hline \multicolumn{7}{|l|}{ Greenwich } \\
\hline Self-confidence workshops, $n$ (\%) & $13(18.3)$ & $51(71.8)$ & $1(1.4)$ & $6(8.5)$ & 0 & 71 \\
\hline Borough population, $n$ 1000s (\%) & $20.8(11.8)$ & $132.6(75.2)$ & $13.9(7.9)$ & $3.8(2.2)$ & $5.3(3.0)$ & 176.4 \\
\hline \multicolumn{7}{|l|}{ Lambeth } \\
\hline Self-confidence workshops, $n$ (\%) & $15(29.4)$ & $24(47.1)$ & $8(15.7)$ & $2(3.9)$ & $2(3.9)$ & 51 \\
\hline Borough population, $n$ 1000s (\%) & $40.4(18.0)$ & $157.5(70.1)$ & $12.9(5.7)$ & 7.5 (3.3) & $6.4(2.8)$ & 224.6 \\
\hline \multicolumn{7}{|l|}{ Lewisham } \\
\hline Self-confidence workshops, $n$ (\%) & $44(32.4)$ & $65(47.8)$ & $15(11.0)$ & $8(5.9)$ & $4(2.9)$ & $138^{\mathrm{C}}$ \\
\hline Borough population, n 1000s (\%) & $42.1(20.3)$ & $140.6(67.8)$ & $12.7(6.1)$ & $6.1(2.9)$ & $6.1(2.9)$ & 207.5 \\
\hline \multicolumn{7}{|l|}{ Croydon } \\
\hline Self-confidence workshops, $n$ (\%) & $26(15.8)$ & $97(58.8)$ & $25(15.2)$ & $14(8.5)$ & $3(1.8)$ & $166^{\mathrm{b}}$ \\
\hline Borough population, $n$ 1000s (\%) & $38.0(14.1)$ & $180.1(66.9)$ & $37.6(14.0)$ & $7.9(2.9)$ & $5.6(2.1)$ & 269.1 \\
\hline \multicolumn{7}{|l|}{ Wandsworth } \\
\hline Self-confidence workshops, $n$ (\%) & $5(8.1)$ & $50(80.6)$ & $5(8.1)$ & $1(1.6)$ & $1(1.6)$ & $62^{\mathrm{b}}$ \\
\hline Borough population, n 1000s (\%) & $16.1(6.8)$ & $192.1(80.8)$ & $17.5(7.4)$ & $5.5(2.3)$ & $6.4(2.7)$ & 237.8 \\
\hline \multicolumn{7}{|l|}{ Bexley } \\
\hline Self-confidence workshops, $n$ (\%) & $5(7.4)$ & $58(85.3)$ & $4(5.9)$ & $1(1.5)$ & 0 & 68 \\
\hline Borough population, $n$ 1000s (\%) & $8.9(5.0)$ & $156.1(87.9)$ & $7.4(4.2)$ & $2.4(1.4)$ & $2.7(1.5)$ & 177.5 \\
\hline \multicolumn{7}{|l|}{ Merton/Sutton } \\
\hline Self-confidence workshops, $n$ (\%) & $6(10.7)$ & $35(62.5)$ & $12(21.4)$ & $3(5.4)$ & 0 & 56 \\
\hline Borough population, $n$ 1000s (\%) & $18.1(5.8)$ & $247.7(79.5)$ & $29.1(9.3)$ & $6.3(2.0)$ & $10.4(3.3)$ & 311.5 \\
\hline \multicolumn{7}{|l|}{ Kingston upon Thames } \\
\hline Self-confidence workshops, $n$ (\%) & $2(2.8)$ & $47(66.2)$ & $19(26.8)$ & $3(4.2)$ & 0 & 71 \\
\hline Borough population, $n$ 1000s (\%) & $3.0(2.3)$ & $103.7(80.2)$ & $12.5(9.7)$ & $2.5(1.9)$ & $7.6(5.9)$ & 129.3 \\
\hline \multicolumn{7}{|l|}{ Total } \\
\hline Self-confidence workshops, $n(\%)^{\mathrm{d}}$ & $116(17.0)$ & $427(62.8)$ & 89 (13.1) & $38(5.6)$ & $10(1.5)$ & 684 \\
\hline Borough population, $n$ 1000s (\%) & $187.4(10.8)$ & $1310.4(75.6)$ & $143.6(8.3)$ & $42.0(2.4)$ & $50.5(2.9)$ & 1733.7 \\
\hline \multicolumn{7}{|c|}{$\begin{array}{l}\text { a. Office for National Statistics population data for } 16+\text { year olds in } 2007 .^{2} \\
\text { b. Missing ethnicity data for } 1 \text { participant. Percentages adjusted to account for this. } \\
\text { c. Missing ethnicity data for } 2 \text { participants. Percentages adjusted to account for this. } \\
\text { d. Missing ethnicity data for } 4 \text { participants. Percentages adjusted to account for this. }\end{array}$} \\
\hline
\end{tabular}

\begin{tabular}{|c|c|c|}
\hline & $\begin{array}{l}\text { Experimental group } \\
\qquad(n=228), n(\%)\end{array}$ & $\begin{array}{l}\text { Control group } \\
(n=230), n(\%)\end{array}$ \\
\hline Treatment completed ${ }^{\mathrm{a}}$ & $151(66)$ & $226(98)$ \\
\hline Treatment not completed, or protocol violated & $77(34)$ & $4(2)$ \\
\hline \multicolumn{3}{|l|}{ Further description of attendance } \\
\hline Attended & $151(66)$ & $134(58)$ \\
\hline Partial attendance & $12(5)$ & $10(4)$ \\
\hline Did not attend & $59(26)$ & $81(35)$ \\
\hline \multicolumn{3}{|l|}{ Attended workshop intended for opposite arm } \\
\hline Allocated experimental, attended control workshop & $5(2)$ & 0 \\
\hline Allocated control, attended experimental workshop & 0 & $4(2)$ \\
\hline Attended but non-eligible/did not want to participate workshop & $1(0.4)$ & $1(0.4)$ \\
\hline
\end{tabular}


Scores for the GAD-7 and RSES was missing in 56 (25\%) and 57 (25\%) participants respectively in the experimental arm, and both scores were missing in 37 (16\%) of control participants (Table 4).

All differences between the experimental and control arms on the primary (BDI) and secondary outcomes (GAD-7, RSES) were statistically significant (Fig. 2 and Table 4). The BDI scores of the experimental arm were 5.3 points lower compared with the control arm. This corresponds to an adjusted effect size of 0.55 . Scores on GAD-7 were lower by 1.6 points and RSES higher by 1.8 points in the experimental arm. In the per protocol analysis, BDI scores were 7.7 points lower in the experimental arm $(95 \%$ CI -10.1 to -5.4$)$. The missing at random sensitivity analysis suggested that the missing values in the experimental arm would have to average 18-20 points higher to render the difference between the arms to be non-significant, a difference which does not seem plausible (see online Fig. DS1).

There was no evidence of therapist learning effect, with a non-significant decrease of 0.3 points in BDI for each additional workshop run (95\% CI -0.8 to $0.3, P=0.28$ ).

In terms of prognostic factors, a higher baseline BDI was found to predict a higher outcome score (Table 5). Being categorised in an employment group other than paid employment was predictive of a worse outcome, but the only statistically significant difference was between housewife/husband and paid employment. Ethnicity did not predict outcome. However, the treatment effect was moderated by gender. The absolute BDI scores at the 12-week outcome for men in the experimental arm were non-significantly lower by 1.7 points $(95 \% \mathrm{CI}-5.6$ to
2.1) compared with those in the control arm, but women in the experimental arm scored significantly lower on the BDI by 6.4 points $(95 \%$ CI -8.8 to -4.0 ) compared with those in the control arm.

The CSQ was completed by $90 \%$ (136 of 151) of experimental arm workshop attenders. Overall satisfaction with the workshops was very high, with $96 \%$ being mostly or very satisfied, and $96 \%$ mostly or very satisfied with the amount of help received. Most (95\%) said they generally or definitely received the help they wanted; $78 \%$ said most or almost all of their needs had been met, and $98 \%$ would recommend the programme to a friend. The service helped $94 \%$ deal more effectively with their problems and $93 \%$ would return to the service.

\section{Economic analysis}

Workshop cost

The average total intervention cost per person in the experimental arm was $£ 161$ (s.d. $=\mathfrak{E 7 6 )}$ in the base case. In the best case scenario, it was $£ 95$ (s.d. $=£ 27$ ) and in the worst case, it was $\mathfrak{E} 192$ (s.d. $=\mathfrak{E} 110)$.

\section{Service use and costs}

There were no statistically significant cost differences between the arms at baseline or follow-up, with the exception of the intervention cost. The total costs at baseline were $£ 1041$ in the experimental arm and $\mathfrak{1 0 5 0}$ in the control arm. The biggest contributor to total costs were hospital costs, although GPs were

Table 4 Unadjusted summary statistics and adjusted mean differences at follow-up for primary and secondary outcome measures

\begin{tabular}{|c|c|c|c|c|c|c|}
\hline & \multicolumn{2}{|c|}{ Experimental group } & \multicolumn{2}{|c|}{ Control group } & \multirow{2}{*}{$\begin{array}{c}\text { Adjusted mean } \\
\text { difference }(95 \% \mathrm{Cl})\end{array}$} & \multirow[b]{2}{*}{$P$} \\
\hline & Unadjusted mean (s.d.) & $n$ & Unadjusted mean (s.d.) & $n$ & & \\
\hline \multicolumn{7}{|c|}{ Beck Depression Inventory } \\
\hline Baseline & $28.9(10.1)$ & 228 & $29.7(10.8)$ & 230 & & \\
\hline 3 months & $19.0(13.3)$ & 180 & $25.4(11.8)$ & 202 & $-5.3(-7.6$ to -2.9$)$ & 0.001 \\
\hline \multicolumn{7}{|c|}{ Generalised Anxiety Disorder-7 } \\
\hline Baseline & $11.7(5.0)$ & 228 & $11.8(5.2)$ & 230 & & \\
\hline 3 months & $8.6(6.0)$ & 172 & $10.5(5.3)$ & 193 & $-1.6(-2.8$ to -0.4$)$ & 0.015 \\
\hline \multicolumn{7}{|c|}{ Rosenberg Self-Esteem Scale } \\
\hline Baseline & $11.9(4.3)$ & 228 & $12.0(4.7)$ & 230 & & \\
\hline 3 months & $14.6(5.0)$ & 171 & $12.9(4.8)$ & 193 & $1.8(0.9$ to 2.7$)$ & 0.003 \\
\hline
\end{tabular}
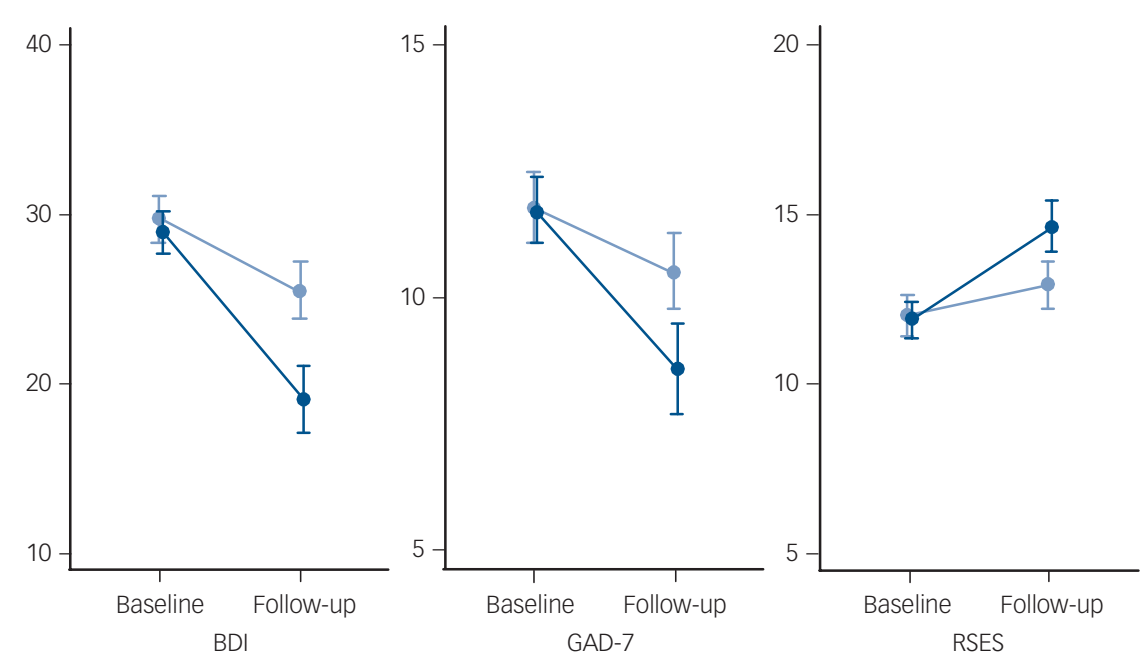

$\longrightarrow$ Experimenta

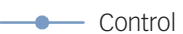

Fig. 2 Unadjusted mean $(95 \% \mathrm{CI})$ profile plots of Beck Depression Inventory (BDI), seven-item Generalised Anxiety Disorder (GAD-7) and Rosenberg Self-Esteem Scale (RSES) 


\begin{tabular}{|c|c|c|c|c|}
\hline Variable & Unadjusted $\beta(95 \% \mathrm{Cl})$ & F-test $P$ & Adjusted $\beta^{a}(95 \% \mathrm{Cl})$ & $F$-test $P$ \\
\hline $\begin{array}{l}\text { Treatment arm, experimental } \\
\text { Treatment arm: experimental, men } \\
\text { Treatment arm: experimental, women }\end{array}$ & $\begin{array}{c}-5.9(-8.7 \text { to }-3.1) \\
- \\
-\end{array}$ & $<0.001$ & $\begin{array}{c}- \\
-1.7(-5.6 \text { to } 2.1) \\
-6.4(-8.8 \text { to }-4.0)\end{array}$ & $\begin{aligned} & 0.37 \\
< & 0.001\end{aligned}$ \\
\hline Baseline BDI & $0.8(0.7$ to 0.9$)$ & $<0.001$ & $0.8(0.7$ to 0.9$)$ & $<0.001$ \\
\hline Ethnicity, other & $-1.4(-4.3$ to 1.5$)$ & 0.25 & $-0.9(-3.5$ to 1.7$)$ & 0.41 \\
\hline Women & $-2.5(-6.7$ to 1.6$)$ & 0.16 & - & \\
\hline Baseline GAD-7 & $1.1(0.9$ to 1.4$)$ & $<0.001$ & - & \\
\hline Baseline RSES & $-1.1(-1.5$ to -0.8$)$ & $<0.001$ & - & \\
\hline Age & $-0.02(-0.11$ to 0.08$)$ & 0.65 & - & \\
\hline $\begin{array}{l}\text { Education } \\
\text { A-level } \\
\text { GNVQ } \\
\text { University degree } \\
\text { Other }\end{array}$ & $\begin{array}{l}-2.7(-7.3 \text { to } 1.8) \\
2.0(-3.8 \text { to } 7.8) \\
-2.5(-5.2 \text { to } 0.3) \\
3.8(-3.0 \text { to } 10.7)\end{array}$ & 0.035 & $\begin{array}{l}- \\
- \\
- \\
-\end{array}$ & \\
\hline $\begin{array}{l}\text { Baseline employment group } \\
\text { Volunteer work } \\
\text { Unemployed } \\
\text { Student } \\
\text { Housewife/husband } \\
\text { Retired } \\
\text { Long-term sick/disabled }\end{array}$ & $\begin{array}{c}4.5(-1.4 \text { to } 10.3) \\
4.4(0.2 \text { to } 8.5) \\
5.8(-4.5 \text { to } 16.1) \\
8.0(2.4 \text { to } 13.5) \\
1.9(-3.9 \text { to } 7.7) \\
5.9(0.1 \text { to } 11.7)\end{array}$ & $<0.001$ & $\begin{array}{c}2.1(-2.4 \text { to } 6.5) \\
2.4(-1.1 \text { to } 5.9) \\
3.7(-4.8 \text { to } 12.2) \\
5.7(0.3 \text { to } 11.0) \\
0.8(-3.2 \text { to } 4.7) \\
2.1(-3.6 \text { to } 7.8)\end{array}$ & 0.054 \\
\hline Previous psychological treatment & 4.8 (1.3 to 8.3$)$ & 0.001 & - & \\
\hline Baseline EQ-5D utility score (per 0.1 units) & $-1.7(-2.1$ to -1.2$)$ & $<0.001$ & - & \\
\hline
\end{tabular}

the most commonly used service, with $45 \%$ in the experimental arm and 53\% in the control arm reporting contacts at baseline. (See online Tables DS2-DS4 for full service use and cost.)

At follow-up, total costs were $\mathfrak{E} 834$ in the experimental arm

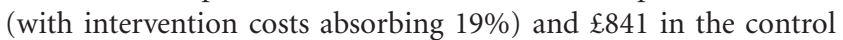
arm. In the experimental arm, the cost of hospital services had decreased significantly over time. The reduction in primary care costs was significant in the control arm at the $90 \%$ level.

At baseline, about $20 \%$ of participants across both arms were in contact with counsellors and with mental health services such as psychologists. Although the proportion in contact with specialist mental health services reduced (non-significantly) by about $5 \%$ in each arm at follow-up, the proportion in contact with counsellors remained the same.

Average total costs for women were $\mathfrak{E} 1136$ (s.d. $=\mathfrak{£} 173$ ) at baseline and $\mathfrak{E} 849$ (s.d. $=\mathfrak{E} 88$ ) at follow-up, whereas for men, these were $\mathfrak{E} 661$ (s.d. $=£ 911)$ and $\mathfrak{k} 784$ (s.d. $=\mathfrak{E} 1260)$ respectively. There were no statistically significant gender differences for the full sample, or between arms.

\section{Cost-effectiveness and cost-utility analyses}

Table 6 shows the average QALY gain, improvement in BDI and additional DFDs for the participants in the cost-effectiveness analysis sample. The QALY gain was small in both arms and there was no statistically significant difference.
Figure 3 shows the probability that the intervention would be considered cost-effective for various levels of WTP for a unit improvement in BDI and QALYs gained respectively, under base case, best case and worst case assumptions.

In the base case scenario, the probability that the intervention is cost-effective in terms of BDI improvement is $30 \%$ at a WTP of zero. This increases to $80 \%$ at a WTP of $£ 30$ and to over $99 \%$ at $\mathfrak{E} 70$.

Given the small QALY gain, the results of the cost-utility analysis are less promising. In the base case, the probability that the intervention is cost-effective first reaches a 50:50 chance at a WTP of $£ 19500$ and does not exceed a probability of $56 \%$ even at higher values of WTP.

Figure 3 also shows the results of the sensitivity analysis (total costs adjusted using intervention costs estimated as best and worst case). The results do not change dramatically, with the relative shape of the curve remaining the same and the expected impact on the probability of cost-effectiveness being small. Full details can be found in online Table DS5.

Looking at DFDs, findings from the cost-effectiveness analysis reflect the difference in BDI outcome (Fig. 4). In the base case, at a WTP of $£ 14$ per DFD, the probability that the intervention is considered cost-effective is $90 \%$. The subgroup analysis by gender (Fig. 5) suggests that the intervention is more likely to be considered cost-effective for men at lower values of WTP, with a

Table 6 Health economic analyses of outcomes

\begin{tabular}{|c|c|c|c|c|c|c|}
\hline & \multicolumn{2}{|c|}{ Control group } & \multicolumn{2}{|c|}{ Experimental group } & \multicolumn{2}{|c|}{ Group difference } \\
\hline & Mean (s.d.) & $n$ & Mean (s.d.) & $n$ & Mean (Cl) & $P$ \\
\hline Change in Beck Depression Inventory score & $3.51(8.32)$ & 201 & $9.47(10.91)$ & 179 & 5.96 (4.01 to 7.91$)$ & $<0.001$ \\
\hline Additional depression-free days & $9.62(24.99)$ & 201 & $28.85(31.16)$ & 179 & 19.23 (13.56 to 24.90$)$ & $<0.001$ \\
\hline Quality-adjusted life-year gain & $0.010(0.61)$ & 193 & $0.007(0.06)$ & 172 & $0.003(-0.01$ to 0.012$)$ & 0.582 \\
\hline
\end{tabular}




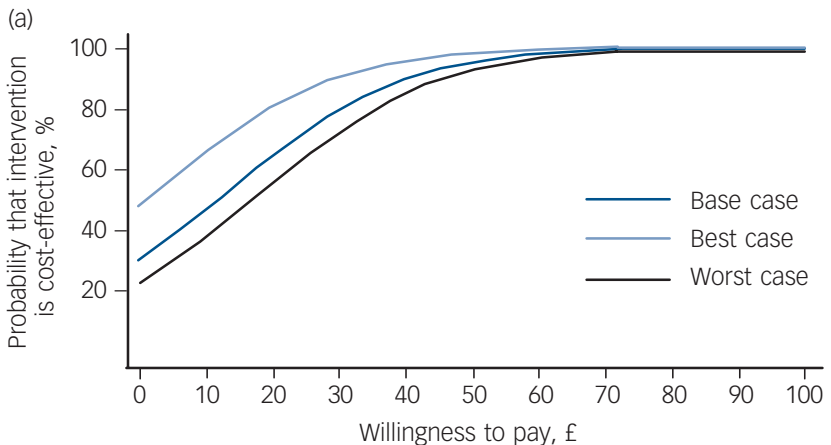

(b)

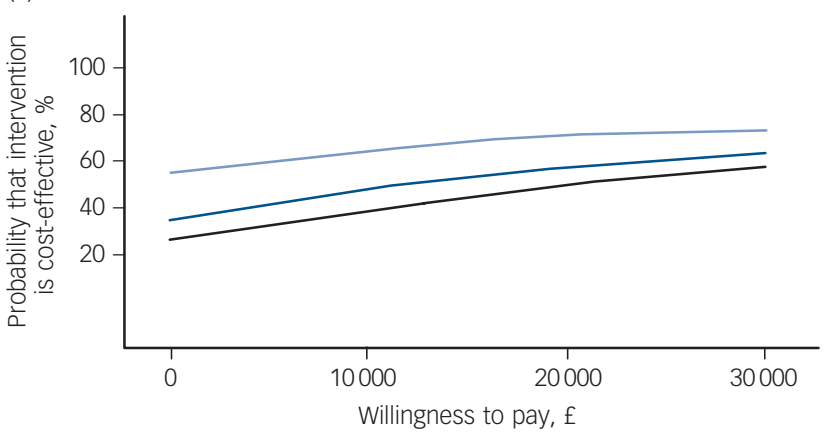

Fig. 3 Cost-effectiveness acceptability curves for changes in (a) Beck Depression Inventory measure and (b) quality-adjusted life-years for different levels of willingness to pay, with sensitivity analysis.

$60 \%$ chance for men and only a $25 \%$ chance of cost-effectiveness for women at WTP of zero. However, with increasing values of WTP, the chance of cost-effectiveness for women increases steeply and exceeds that for men at a WTP of about $£ 15$. This reflects the significantly higher BDI improvement and (non-significantly) higher costs for women compared with men.

\section{Discussion}

This RCT shows that at 12 weeks, 1-day psychoeducational selfconfidence workshops are clinically effective at improving depression in a community sample recruited from areas of differing deprivation. Additionally, the workshops were effective at reducing levels of anxiety and increasing self-esteem. There was a differential effect of gender on depression outcome, whereby women benefited more from the workshops than men. The workshops attracted difficult to engage groups. A quarter of the participants had not previously consulted their GP for depression. A higher proportion of individuals from BME groups also participated than would be expected from the local population distribution.

The economic analysis found no significant differences in baseline total support costs between the experimental and control arms. Based on depressive symptoms (BDI) and the related measure of depression-free days, the intervention has a high probability of being cost-effective, given a significant improvement in BDI in the experimental arm over and above the improvement seen in the control arm.

\section{Strengths and weaknesses}

There are several strengths to this study. This larger, rigorously conducted clinical trial extends the work of a preliminary RCT evaluating self-confidence workshops ${ }^{14}$ and shows that workshops

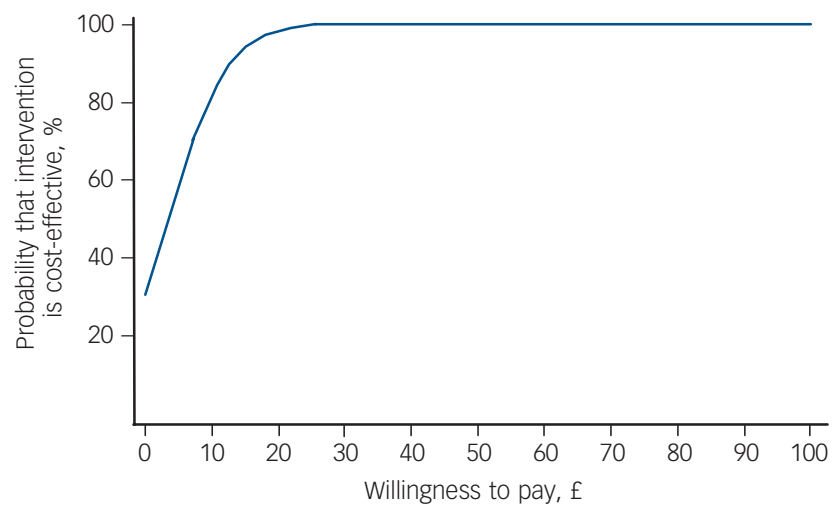

Fig. 4 Cost-effectiveness acceptability curves for additional depression-free days for different levels of willingness to pay.

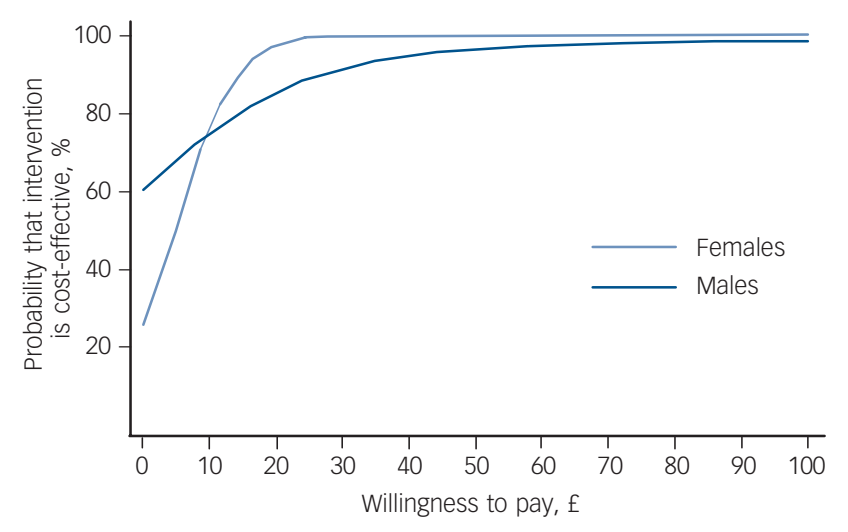

Fig. 5 Cost-effectiveness acceptability curves for additional depression-free days for different levels of willingness to pay: subgroup analysis by gender.

targeted at people with depression attained an effect size of 0.55 on the BDI. This compares favourably with the mean effect size of 0.31 that has been found in a meta-analysis of RCTs of psychological treatments for depression in primary care. ${ }^{37}$

The study had a large sample size and was multicentre, with the workshops covering some of the most deprived (e.g. Greenwich, Lambeth) and least deprived (e.g. Kingston upon Thames, Merton/Sutton) boroughs in England. The results are therefore likely to be generalisable to populations with varying levels of deprivation.

A major strength was its accessibility. Using a self-referral system to attract members of the public with depression to the workshops was very successful, with the introductory talks oversubscribed in most boroughs. Importantly, $25 \%$ of the participants had never consulted their GP about their psychological problems, presumably either not seeing their GP about their depression or not raising their depression if they did consult. This finding is all the more remarkable given the common difficulties in engaging people with depression in treatment. ${ }^{2}$ This supports previous studies showing that providing an accessible alternative route can help improve help-seeking for depression by the public. $^{38}$

Notably, there was a higher proportion (32\%) of participants from BME groups than expected. Participants represented 1.5 times the BME population in five boroughs and twice the Asian population in three boroughs. This is remarkable given the reluctance of BME communities to consult their GPs for 
psychological problems and the low number using psychological services. ${ }^{6}$ However, it should be acknowledged that the borough figures cited only reflect the gender and ethnic breakdown and not necessarily the characteristics of the depressed populations. Of note though, was that there were no ethnic differences in outcome. It should also be noted that Black participants were not differentiated into African or Caribbean.

A further strength was that the use of non-diagnostic labels attracted a large proportion (63\%) of people with depression. This supports previous findings indicating that the use of nondiagnostic labels, such as 'self-confidence' rather than depression or 'sleep' rather than insomnia, can be important in engaging groups who may prefer not to medicalise mental health problems. ${ }^{38}$ The general public have been shown to conceptualise depressive symptoms as 'problems of living' rather than symptoms of a mental illness. ${ }^{39}$ It may be that the offer of psychological intervention with a non-medical label provided an alternative route to psychological help more congruent with the health beliefs of the public.

There are several methodological strengths of this trial. Stratified randomisation was used, pre-randomisation allocation concealment was maintained, the statistician masked prior to analysis, and analyses were performed on an ITT basis.

Some limitations were that only self-report measures were used, so that the assessment of depression is reliant on this rather than on clinical interview. However, clinical diagnostic interviews for depression were not feasible given the large community samples. The workshops also attracted quite a high proportion of graduates $(44 \%)$, possibly biasing the results.

Another limitation was that the attrition rate at the beginning of the study was $32 \%$ but was improved to $16 \%$ so that data for $83 \%$ of participants were available at follow-up. However, a sensitivity analysis for the missing at random assumption made by multiple imputation methods indicated that the missing participants would have had to have scored incredibly higher or lower compared with those who did return data in order to render the result non-significant. The follow-up rate for the control arm was higher than for the experimental arm, probably in part because the control arm completed their questionnaires when they arrived for their intervention rather than 12 weeks after the experimental arm workshop.

Other weaknesses were that treatment quality and fidelity were assessed only through observation of two workshops run by newly trained leaders. This workshop seemed to appeal to women much more than men, and also seemed to be more helpful to women. Finally, limitations were a lack of a placebo control so that expectancy was not controlled, and follow-up data were only collected after 3 months.

Although the cost-effectiveness findings are encouraging for the depression-specific measure, the intervention is unlikely to be considered cost-effective in terms of QALYs, given the small QALY improvement. Additionally, QALYs are a generic measure of health-related quality of life that facilitates comparability across interventions and conditions and it has been argued ${ }^{40}$ that they may not be sensitive enough to assess changes resulting from interventions targeting mental, rather than physical, health. Only one of the five EQ-5D dimensions relates directly to mental health (anxiety/depression), so in this study a very large improvement in this domain would have been required to generate a significant improvement in the overall measure.

\section{Comparison with other studies}

As this psychoeducational intervention differs substantially from other programmes for depression, particularly in terms of content and the 1-day format, direct comparisons are difficult. This section will therefore focus on the comparison of effectiveness of this intervention with other primary care interventions for depression.

The effect size of these self-confidence workshops plus booster compared favourably with other primary care interventions. It is higher than that of a traditional 12-session 'Coping with Depression' course, which a meta-analysis found to have a mean effect size of only $0.28 .{ }^{41}$ It also compares well with computerised CBT for depressive symptoms where a meta-analysis found an effect size of $0.32 .^{42}$ Similarly, collaborative care which involves shared care between the primary care physician, psychiatrist and psychologist, with the patient involved in making decisions has been found to be effective. However, the overall effect size for this approach was reported as $0.20-0.29 .^{43}$

The self-confidence workshop intervention compares very favourably with self-help without additional guidance, where an effect size of 0.28 was found in a meta-analysis. ${ }^{44}$ However, guided self-help, which offers CBT materials with some support from a health professional, fared better, with an effect size of $0.80 .{ }^{45}$

One may speculate on reasons for the comparative effectiveness of the workshops. One is that the group support offered in the workshops could have been important, especially when compared with computerised CBT. Collaborative care could be as effective or ineffective as the quality of local services available. It is interesting that the small group depression course had a lower effect size but this could be attributed to differences in the course as the selfconfidence workshops used cognitive as well as behavioural methods. Finally, the larger effect size obtained by guided self-help may be the result of being able to provide detailed follow-up of individual goals over several weeks, which is not possible with a 1-day programme.

The cost-effectiveness findings based on BDI improvement compare favourably with computerised CBT for anxiety and depression in primary care. ${ }^{46}$ From a societal perspective (including lost employment) the base case scenario for computerised CBT showed a 14\% probability that the intervention was cost-effective at a WTP of zero. This increased to over $80 \%$ at WTP values over $£ 40$ and to over $90 \%$ at a WTP of $£ 80$. In comparison, the psychoeducational workshops showed a higher probability of cost-effectiveness of $30 \%$ at a WTP of zero, which

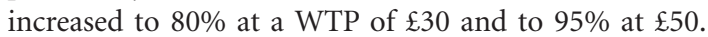

However, when looking at DFDs, the probability that the computerised CBT intervention would be considered costeffective was $80 \%$ at a WTP of $£ 5$ per DFD compared with only $54 \%$ for the workshops. Further, the rate at which the probability of cost-effectiveness increases as WTP rises is slower for the workshops than for computerised CBT. It has to be noted, however, that our estimate of DFDs is fairly crude. It is based on only two values for BDI, whereas in the computerised CBT study, BDI data were collected at five time points over a 6-month period.

The current trial compares favourably with a study comparing CBT with usual care and talking therapy for older people, ${ }^{47}$ which found a cost of $£ 120$ per point reduction in BDI and a $90 \%$ probability that CBT would be considered cost-effective at a

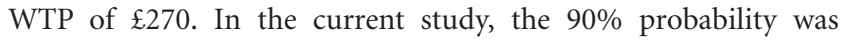
reached at a WTP of only $£ 40$ in the base case.

Comparison with studies that do not present cost-effectiveness acceptability curves is not straightforward, but cost per DFD is a commonly reported comparable measure. A review of collaborative care interventions in primary care reported costs per DFD of between $\$ 20$ and $\$ 24,{ }^{48}$ equivalent to approximately $\mathfrak{E} 14$ (using purchasing power parity data for 2010 prices; http://stats.oecd.org). At this value of WTP, the probability that the self-confidence 
workshops would be considered cost-effective is $80 \%$ in the base case, suggesting that the cost per DFD of self-confidence workshops is broadly the same as for collaborative care interventions.

\section{Service implications}

The self-confidence workshops approach could well help the undertreatment of depression. It could provide a viable and effective alternative way for the public to directly access a brief and acceptable psychological intervention for depression, by offering early intervention to those who are reluctant to seek help from their GPs, such as those from BME communities, in areas of varying social deprivation. It could also help circumvent the common problem of underdetection of depression in primary care. $^{3}$

Given the dearth of evidence on the cost-effectiveness of interventions for depression, this study is a valuable contribution to the evidence base. The intervention is relatively cheap to provide, at around $£ 161$ per participant, and there is no associated increase in other support costs. It should therefore be considered a cost-effective way of engaging people who receive little in terms of other support, despite high levels of distress. It is a promising option that could further the aims of the IAPT services in the UK.

\section{Future research}

It would be helpful to replicate these findings and investigate the effectiveness and cost-effectiveness of these day-long selfconfidence workshops using a placebo control workshop where participants attend for a day, but are given an alternative treatment for depression or alternatively a control condition where expectancy is controlled, such as a health education programme. It would also be useful to examine the longer-term effectiveness of these workshops over at least a year, even though a naturalistic follow-up indicated that the effects were maintained after 2 years by those with depression. ${ }^{15}$

An examination of which methods participants found particularly useful and actually put into practice would also be informative. These components could be studied further to see which mediate the treatment effect in order to better tailor future workshops. It would be useful to run workshops in areas where there are figures for local psychiatric morbidity. It would also be useful to attract non-graduate difficult-to-engage groups to see whether this affects results.

In this study, gender affected uptake and outcome. Although the prevalence of depression is higher among women, women are more likely to perceive a need for mental healthcare than men. ${ }^{49}$ Men may be more reluctant, possibly because of male gender-role expectations and gender-related health concepts which hinder help-seeking. ${ }^{49}$ Men with depression have also been found to present fewer symptoms and cope differently. ${ }^{50}$ In this study, we found that men tended more often to have consulted their GPs with mild depression, which is often difficult to detect in primary care. Further work to develop interventions that will both appeal and effectively help men with depression would be important.

Finally, further research regarding the health beliefs of the general public could lead to the use of acceptable 'labels' that would appeal to men and women, which could in turn lead to the development of more large-scale psychological health interventions for the public.

\section{Funding}

This paper presents independent research funded by the National Institute for Health Research (NIHR) under its Research for Patient Benefit (RfPB) Programme (Grant Reference
Number PB-PG-1207-15154). The views expressed are those of the authors and not necessarily those of the NHS, the NIHR or the Department of Health. J.S.L.B., A.T. and U.S. are partly funded by the NIHR Mental Health Biomedical Research Centre (BRC) at the Institute of Psychiatry and South London and Maudsley Foundation Trust. J.B. and E.M.B. are partly funded by NIHR Programme Grants for Applied Research.

\section{Acknowledgements}

Other members of the CLASSIC trial group: Jeanette Augustin, Marta Buszewicz, Jed Boardman and Christine Longinotti.

Linda Horrell, DipPsych, Psychology Department, Kimberley A. Goldsmith, MPH Biostatistics Department, André T. Tylee, MD, Health Services and Population Research, Ulrike H. Schmidt, PhD, Section of Eating Disorders, Caroline L. Murphy, MSc, Clinical Trials Unit, Institute of Psychiatry, King's College London; Eva-Maria Bonin, MA, Personal Social Services Research Unit, London School of Economics and Political Science; Jennifer Beecham, PhD, Personal Social Services Research Unit, London School of Economics and Political Science, and University of Kent; Joanna Kelly, MSC, Clinical Trials Unit, Shriti Raikundalia, MSC, Psychology Department, Institute of Psychiatry, King's College London; June S. L. Brown, PhD, Psychology Department, Institute of Psychiatry, King's College London, UK

Correspondence: June S. L. Brown, Psychology Department (PO77), Institute of Psychiatry, De Crespigny Park, London SE5 8AF, UK. Email: june.brown@kcl.ac.uk First received 4 Oct 2012, final revision 11 Apr 2013, accepted 24 Jun 2013

\section{References}

1 World Health Organization. The Global Burden of Disease: 2004 Update. WHO, 2008

2 Collins KA, Westra HA, Dozois DJA, Burns DD. Gaps in accessing treatment for anxiety and depression: challenges for the delivery of care. Clin Psychol Rev 2004; 24: 583-616.

3 Bebbington PE, Meltzer $\mathrm{H}$, Brugha TSA, Farrell M, Jenkins $\mathrm{R}$, Ceresa $\mathrm{C}$, et al. Unequal access and unmet need: neurotic disorders and the use of primary care services. Psychol Med 2000; 30: 1359-67.

4 Riedel-Heller SG, Matschinger H, Angermeyer MC. Mental disorders - who and what might help? Soc Psychiatry Psychiatr Epidemiol 2005; 40: 167-74.

5 Layard R. The case for psychological treatment centres. BMJ 2006; 332: 1030-2.

6 Williams P, Turpin G, Hardy G. Clinical psychology service provision and ethnic diversity within the UK: a review of the literature. Clin Psychol Psychother 2006; 13: 324-38.

7 Clark DM, Layard R, Smithies R, Richards DA, Suckling R, Wright B. Improving access to psychological therapy: Initial evaluation of two UK demonstration sites. Behav Res Ther 2009; 47: 910-20.

8 DeRubeis RJ, Hollon SD, Amsterdam JD, Shelton RC, Young PR, Salomon RM, et al. Cognitive therapy vs. medications in the treatment of moderate to severe depression. Arch Gen Psychiatry 2005; 62: 409-16.

9 McDermut W, Miller I, Brown R. The efficacy of group psychotherapy for depression: a meta-analysis and review of the empirical research. Clin Psychol Sci Pract 2001; 8: 98-116.

10 National Collaborating Centre for Mental Health. Depression: The NICE Guideline on the Treatment and Management of Depression in Adults (Updated Edition) (National Clinical Practice Guideline 90). British Psychological Society and Royal College of Psychiatrists, 2009.

11 White J, Keenan M, Brooks N. Stress control: a controlled comparative investigation of large group therapy for generalized anxiety disorder. Behav Psychother 1992; 20: 97-114.

12 Brown JSL, Cochrane R, Hancox T. Large scale stress management workshops for the general public: a controlled evaluation. Behav Cog Psychother 2000; 28: 139-51.

13 Watkins E, Elliott S, Stanhope N, Button J, Williams J, Brown JSL. Meeting the needs for psychological treatment of people with common mental disorders: an exploratory study. J Ment Health 2000; 9: 445-56.

14 Brown JSL, Elliott SA, Boardman J, Ferns J, Morrison J. Meeting the unmet need for depression services with psycho-educational self-confidence workshops: preliminary report. Br J Psychiatry 2004; 185: 511-5.

15 Brown JSL, Elliott SA, Boardman J, Andiappan M, Landau S, Howay E. Can the effects of a 1-day CBT psychoeducational workshop on self-confidence be maintained after 2 years? A naturalistic study. Depress Anxiety 2008; 25 632-40.

16 Leeser R. English Indices of Deprivation 2010: A London Perspective. Greater London Authority, 2011. 
17 Beck AT, Steer RA, Brown GK. Manual for the Beck Depression Inventory-II. Psychological Corporation, 1996.

18 Fennell M. Overcoming Low Self-Esteem: A Self-Help Guide using Cognitive Behavioural Techniques. Robinson, 1999.

19 Beck AT, Ward CH, Mendelson M, Mock J, Erbaugh J. An inventory for measuring depression. Arch Gen Psychiatry 1961; 4: 561-71.

20 Lave J, Frank R, Schulberg H, Kamlet M. Cost-effectiveness of treatments for major depression in primary care practice. Arch Gen Psychiatry 1998; 55: 645-51.

21 Spitzer RL, Kroenke K, Williams JBW, Lowe B. A brief measure for assessing generalized anxiety disorder: the GAD-7. Arch Intern Med 2006; 166: 1092-7.

22 Blascovich J, Tomaka J. Measures of self-esteem. In Measures of Personality and Social Psychological Attitudes (3rd edn) (eds JP Robinson, PR Shaver LS Wrightsman): 115-60. Institute for Social Research, 1993.

23 EuroQol Group. EuroQol - a new facility for the measurement of health related quality of life. Health Policy 1990; 16: 199-208.

24 Dolan P. Modeling valuations for EuroQol health states. Med Care 1997; 35 1095-108.

25 Beecham J, Knapp M. Costing psychiatric interventions. In Measuring Mental Health Needs (2nd edn) (ed. G Thornicroft): 200-24. Gaskell, 2001.

26 Attkisson CC, Zwick R. The client satisfaction questionnaire. Psychometric properties and correlations with service utilization and psychotherapy outcome. Eval Prog Plan 1982; 5: 233-7.

27 Larsen DL, Attkisson CC, Hargreaves WA, Nguyen TD. Assessment of client/ patient satisfaction: development of a general scale. Eval Prog Plan 1979; 2 197-207.

28 Office for National Statistics. Ethnic Groups by Broad Ethnicity, Age and Gender. ONS, 2007.

29 Royston P. Multiple imputation of missing values. Stata J 2004; 4: 227-41.

30 Little RJA, Rubin DB. Statistical Analysis with Missing Data. John Wiley, 1987.

31 White IR, Carpenter J, Horton NJ. Including all individuals is not enough: lessons for intention-to-treat-analysis. Clin Trials 2012; 9: 396-407.

32 Curtis L. Unit Costs of Health and Social Care 2011. Personal Social Services Research Unit, University of Kent, 2011.

33 Bonin E-M, Beecham J. Costing multi-site, group-based CBT workshops. In Unit Costs of Health \& Social Care 2012 (ed. L Curtis): 17-21. Personal Social Services Research Unit, University of Kent, 2012.

34 Zellner A. An efficient method of estimating seemingly unrelated regression equations and tests for aggregation bias. J Am Stat Assoc 1962; 57: 348-68.

35 Hoch JS, Briggs AH, Willan AR. Something old, something new, something borrowed, something blue: a framework for the marriage of health econometrics and cost-effectiveness analysis. Health Econ 2002; 11: 415-30.

36 van Hout BA, Al MJ, Gordon GS, Rutten FF. Costs, effects and C/E-ratios alongside a clinical trial. Health Econ 1994; 3: 309-19.
37 Cuijpers $P$, van Straten A, van Schaik A, Andersson G. Psychological treatment of depression in primary care: a meta-analysis. $\mathrm{Br} J \mathrm{Jen}$ Pract 2009; 59: e51-60.

38 Brown JSL, Boardman J, Whittinger N, Ashworth M. Can a self-referral system help improve access to psychological treatments? Br J Gen Pract 2010; 60: $365-71$

39 Cornford CS, Hill A, Reilly J. How patients with depressive symptoms view their condition: a qualitative study. Fam Pract 2007; 24: 358-64.

40 Knapp M, Mangalore R. "The trouble with QALYS ...". Epidemiol Psichiatr SOC 2007; 16: 289-93.

41 Cuijpers P, Muñoz RF, Clarke GN, Lewinsohn PM. Psychoeducational treatment and prevention of depression: the "coping with depression" course thirty years later. Clin Psychol Rev 2009; 29: 449-58.

42 Spek V, Cuijpers P, Nyklicek I, Riper H, Keyzer J, Pop V. Internet-based cognitive behaviour therapy for symptoms of depression and anxiety: a meta-analysis. Psychol Med 2007; 37: 319-28.

43 Katon W, Guico-Pabia JG. Improving quality of depression care using organized systems of care: a review of the literature. $\mathrm{Pr}$ Care Companion CNS Disord 2011; 13: e1-8.

44 Cuijpers $\mathrm{P}$, Donker $\mathrm{T}$, Johansson R, Mohr DC, van Straten A, Andersson G. Self-guided psychological treatment for depressive symptoms: a metaanalysis. PloS One 2011; 6: e21274.

45 Gellatly J, Bower P, Hennessy S, Richards D, Gilbody S, Lovell K. What makes self-help interventions effective in the management of depressive symptoms? Meta-analysis and meta-regression. Psychol Med 2007; 37: 1217-28.

46 McCrone P, Knapp M, Proudfoot J, Ryden C, Cavanagh K Shapiro DA, et al. Cost-effectiveness of computerised cognitive-behavioural therapy for anxiety and depression in primary care: randomised controlled trial. Br J Psychiatry 2004; 185: 55-62.

47 Holman AJ, Serfaty MA, Leurent BE, King MB. Cost-effectiveness of cognitive behaviour therapy versus talking and usual care for depressed older people in primary care. BMC Health Serv Res 2011; 11: 33.

48 van Steenbergen-Weijenburg KM, van der Feltz-Cornelis CM, Horn EK, van Marwijk HW, Beekman AT, Rutten FF, et al. Cost-effectiveness of collaborative care for the treatment of major depressive disorder in primary care. A systematic review. BMC Health Serv Res 2010; 10: 19.

49 Moeller-Leimkuehler AM. Barriers to help-seeking by men: a review of sociocultural and clinical literature with particular reference to depression. J Affect Disord 2002; 71: 1-9.

50 Angst J, Gamma A, Gastpar M, Lepine J-P, Mendlewicz J, Tylee A. Gender differences in depression: Epidemiological findings from the European DEPRES I and II studies. Eur Arch Psychiatry Clin Neurosci 2002; 252: 201-9. 